\title{
A message for 2019
}

\author{
Naomichi Matsumoto Editor-in-Chief ${ }^{1,2}$
}

Received: 7 February 2019 / Accepted: 7 February 2019 / Published online: 25 April 2019

(c) The Author(s), under exclusive licence to The Japan Society of Human Genetics 2019

The Journal of Human Genetics published 167 papers in 2018. We prepared a special issue describing "Advances in inborn errors of metabolism" in 2018, which was published in February 2019. I would like to express my sincere appreciation to guest editors, Drs. Toshiyuki Fukao and Kimitoshi Nakamura, and all the contributors for their great work. I hope the special issue will attract attention to a broad readership.

In 2018, we celebrated Professor Tasuku Honjo receiving 2018's Nobel Laureate in Physiology or Medicine. His outstanding achievements in discovery of cancer therapy by

$\begin{array}{ll}\text { Catherine M Abbott } & \text { Raphael Bernier } \\ \text { Kiwamu Akagi } & \text { Rui Bi } \\ \text { Ainur Akilzhanova } & \text { Gillian Blue } \\ \text { Masato Akiyama } & \text { Hanno Bolz } \\ \text { Hidenori Akutsu } & \text { Kevin Booth } \\ \text { Branko Aleksic } & \text { Matthew MER Butchbach } \\ \text { Fowzan Alkuraya } & \text { Francesc Calafell } \\ \text { Wolfram Antonin } & \text { Rosario Calderón } \\ \text { Yoshitsugu Aoki } & \text { Cristian Capelli } \\ \text { Yoko Aoki } & \text { Nuria Carrillo } \\ \text { Reiko Arakawa } & \text { Melissa Carter } \\ \text { Takuro Arimura } & \text { Claudia Carvalho } \\ \text { Akira Ashida } & \text { Teodora Chamova } \\ \text { Midori Awazu } & \text { Wei-Chiao Chang } \\ \text { Seiamak Bahram } & \text { Anne Lynn Chang } \\ \text { Meena Balasubramanian } & \text { Stephen Chanock } \\ \text { Bodo Beck } & \text { Gyaneshwer Chaubey } \\ \text { Christian Beetz } & \text { Wei Chen } \\ \text { Minako Beppu } & \text { Hua Chen }\end{array}$

Naomichi Matsumoto

naomat@yokohama-cu.ac.jp

Journal of Human Genetics, Yokohama, Japan

2 Department of Human Genetics, Yokohama City University Graduate School of Medicine, Yokohama, Japan inhibition of negative immune regulation will open up a new strategy of cancer therapy.

In 2019, we are planning a special issue dealing with a new technology in human genetics researches. I look forward to it and also to receiving more exciting manuscripts for the Journal of Human Genetics from all over the world.

Acknowledgements The Editor-in-Chief, on behalf of the Journal of Human Genetics Editorial Board and the Japan Society of Human Genetics, gratefully acknowledges the generous support from peer reviewers in carefully evaluating manuscripts under consideration. The following individuals provided reviews of manuscripts submitted to the Journal of Human Genetics in 2018:

$\begin{array}{ll}\text { I-Ping Chen } & \text { Katsunori Fujii } \\ \text { Peter Chiba } & \text { Akihiro Fujimoto } \\ \text { Takeshi Chiyomaru } & \text { Kaoru Fujinami } \\ \text { Richard Choy } & \text { Masashi Fujita } \\ \text { John Creemers } & \text { Maki Fukami } \\ \text { Yataro Daigo } & \text { Koya Fukunaga } \\ \text { Sumito Dateki } & \text { Manabu Funayama } \\ \text { Iris De Lange } & \text { Tetsushi Furukawa } \\ \text { João RicardoDe Oliveira } & \text { Hiroshi Furukawa } \\ \text { Aidan J. Doherty } & \text { Hiroto Furuta } \\ \text { Krzysztof Domagalski } & \text { Nobuo Fuse } \\ \text { David Duffy } & \text { Rebecca Ganetzky } \\ \text { Yusuke Ebana } & \text { Bo Gao } \\ \text { Thomas Eggermann } & \text { Angelina García } \\ \text { Andrew Engel } & \text { Mohsen Ghadami } \\ \text { Erv Epstein } & \text { Katta Girisha } \\ \text { Hezhi Fang } & \text { Yuichi Goto } \\ \text { Victor Faundes } & \text { Giedre Grigelioniene } \\ \text { Antonella Forlino } & \text { Andrey Grigoriev }\end{array}$


Nobuhiko Haga

Kazushige Hanaoka

Yuichiro Hara

Naoki Harada

Yutaka Harita

Takanori Hashimoto

Jun Hata

Kenichiro Hata

Shin Hayashi

Takeharu Hayashi

Yukiko Hayashi

Keiko Hikino

Akira Hirasawa

Kenji Hirayama

Tomomitsu Hirota

Yuki Hitomi

Susan Holmes

Momoko Horikoshi

Yoshihiro Hotta

Fukiko Ichida

Shingo Ichimiya

Aritoshi Iida

Masashi Ikeda

Shiro Ikegawa

Minako Imamura

Tsuneo Imanaka

Toshiyuki Imasawa

Issei Imoto

Jun Inoue

Ken Inoue

Ituro Inoue

Melita Irving

Mika Ishige

Kotaro Ishikawa

Hiroyuki Ishiura

Minoru Isomura

Kaoru Ito

Tetsuya Ito

Jessie Jacobsen

Fernanda Jehee

Xiaodong Jiao

Masayo Kagami

Yoichiro Kamatani

Naoyuki Kamatani

Tadashi Kaname

Tetsufumi Kanazawa

Tatsuo Kanda

Megan Kane

Atsushi Kaneda

Peter Kannu

Piranit Kantaputra

Mitsuhiro Kato

Kosuke Kato
Yosuke Kawai

Toshitaka Kawarai

Aya Kawasaki

Atsuo Kikuchi

Tadashi Kimura

Ryosuke Kimura

Akinori Kimura

Akira Kinoshita

Shin-ichiro Kitajiri

Kazuma Kiyotani

Hironori Kobayashi

Satoko Kojima

Hiroyuki Kondo

Eric Konnick

Takao Konomoto

Uwe Kornak

Tomoki Kosho

Shinji Kosugi

Ikuyo Kou

Mitsuru Kubota

Tomu Kuchikata

Punna Kunhapan

Hiroki Kurahashi

Yukiko Kuroda

Yoko Kuroki

Kenji Kurosawa

Akira Kurozumi

Shireen Lamande

Tomoko Lee

Beom Hee Lee

Laurence Legeai-Mallet

Hui Li

Chengtao Li

Hong Liu

Zhen Liu

Shiro Maeda

Jun Maeda

Naomasa Makita

Boris Malyarchuk

Hiroshi Manya

Nina Marchi

Christian Marshall

Julian Martinez-Agosto

Tohru Masui

Keiko Matsubara

Nagahide Matsubara

Tatsuo Matsunaga

Masafumi Matsuo

Tohru Matsuura

Shinya Matsuura

Takashi Matsuzaka

Johannes Mayr

Robert McCullumsmith
David Meyre

Daiki Miki

Francisca Millan Zamora

Steven F. Miller

Jusaku Minari

ShinseiMinoshima

Hiroshi Mitsubuchi

Jun Mitsui

Norisato Mitsutake

Fuyuki Miya

Hiroko Miyadera

Noriko Miyake

Toshinobu Miyamoto

Satoru Miyawaki

Seiichi Mori

Naoya Morisada

Takayuki Morisaki

Ian Morison

Hiroyuki Morita

Bernice E Morrow

Hideaki Moteki

Masaaki Muramatsu

Kei Murayama

Koji Muroya

Taisei Mushiroda

Fuji Nagami

Masayoshi Nagao

Koji Nagao

Yuki Nagata

Izumi Naka

Kazuhiko Nakabayashi

Yoko Nakajima

Kimitoshi Nakamura

Koichi Nakanishi

Yukiko Nakano

Hirofumi Nakaoka

Mitsuko Nakashima

Tomohiro Nakayama

Satoshi Narumi

Taeko Naruse

Sadaf Naz

Hoang Nguyen

Tetsuya Niihori

Takenori Niioka

Yasuharu Nishimura

Gen Nishimura

Jo Nishino

Shinya Nishio

Kenya Nishioka

Kazuma Noguchi

Yoshihiro Noguchi

Emiko Noguchi

Ikuya Nonaka
Kandai Nozu

Chikahiko Numakura

Tsutomu Ogata

Tomoo Ogi

Bermseok Oh

Osamu Ohara

Jun Ohashi

Toya Ohashi

Kazutaka Ohi

Seiko Ohno

Yutaka Ohsawa

Tohru Ohta

Kimihiko Oishi

Nobuhiko Okamoto

YoshiyukiOkano

Yasushi Okazaki

Tetsuya Okazaki

Heymut Omran

Osamu Onodera

Yoshihiro Onouchi

Hitoshi Osaka

Masao Ota

Takahiro Otani

Kouichi Ozaki

Takeshi Ozeki

Keiichi Ozono

Karel Pacak

Emanuele Panza

Woong-Yang Park

Sergio Pena

Min-Sheng Peng

Eugene Pergament

Miguel Pericacho

Paolo Peterlongo

Rafal Ploski

Gudrun Rappold

Frank Rauch

Edward Ruiz-Narváez

Yoshiaki Saito

Takeo Saito

Shinji Saitoh

Hirotomo Saitsu

Norio Sakai

Aiko Sakai

Yasunari Sakai

Osamu Sakamoto

Hiroyuki Sakashita

Mark Sands

Renata Santos

Tatsuharu Sato

Youichi Sato

Hideaki Sawai

Aaron Schindeler 
Charles Schwartz

Naohiko Seki

Alok Sharma

A. Eliot Shearer

Wei Shen

Seiji Shibata

Yosuke Shigematsu

Daichi Shigemizu

Takashi Shiina

Haruo Shimazaki

Kenji Shimizu

Atsushi Shimizu

NobuyukiShimozawa

Kouya Shiraishi

David Sillence

You-Qiang Song

YasuyukiSuzuki

Satoshi Suzuki

Kathryn Swoboda

Yasuharu Tabara

Hayato Tada

Go Tajima

Atsushi Tajima
Toshihiro Tajima

Yuji Takahashi

Kyoko Takano

Miho Takaoka

Hiroshi Takashima

Masaki Takayanagi

Kazuki Takeda

Fumihiko Takeuchi

Gen Tamiya

Tiong Tan

Masashi Tanaka

Mariko Taniguchi-Ikeda

Chizu Tanikawa

Marwan Tayah

Gianluca Tedaldi

Micheal To

Katsushi Tokunaga

Shunji Tomatsu

Hidefumi Tonoki

Niels Tørring

Stephen Tsang

Akihito Tsubota

Clare Turnbull
Henna Tyynismaa

Daniela Uehara

Motoko Unoki

Kevin Urayama

Shin-Ichi Usami

Takahito Wada

Keiko Wakui

Fan Wang

Matthew Warman

Atsushi Watanabe

Ryo Watanabe

Yoriko Watanabe

Chen-Chi Wu

Miao Xu

Michael Yaffe

Takashi Yagi

Ryo Yamada

Kenji Yamada

Takanori Yamagata

Toshiyuki Yamamoto

Kayono Yamamoto

Keiko Yamamoto-

Shimojima
Michiko Yamanaka

Kenji Yamashiro

Keiko Yamazaki

Kiyoshi Yanagisawa

Kazuki Yasuda

Kevin Yip

Tohru Yorifuji

Reiko Yoshida

Hiroshi Yoshihashi

Koh-ichiro Yoshiura

Hyeong Gon Yu

Kei Yura

Andreas Zankl

Massimo Zeviani

Asima Zia

Marcella Zollino 